\title{
Long noncoding RNA TUG1 promotes cardiac fibroblast transformation to myofibroblasts via miR-29c in chronic hypoxia
}

\author{
YUN ZHU, ZEZHOU FENG, ZHAO JIAN and YINGBIN XIAO \\ Department of Cardiovascular Surgery, Xinqiao Hospital, Army Medical University, Chongqing 400037, P.R. China
}

Received May 17, 2018; Accepted July 11, 2018

DOI: $10.3892 / \mathrm{mmr} .2018 .9327$

\begin{abstract}
Cardiac fibroblast-myofibroblast transformation (FMT) contributes to the fibrotic deterioration evoked by chronic hypoxia. Growing evidence implicates long noncoding RNAs (lncRNAs) in various types of cardiac physiological and pathological processes, especially in cardiac fibrosis. In the present study, the lncRNA Taurine Upregulated Gene 1 (TUG1), reported as a regulator of hypoxia fibrosis in the lungs, was found to also be an important regulator of cardiac FMT. Specifically, the possible role of TUG1 in cardiac FMT and fibrosis under chronic hypoxia was investigated. It was revealed that the degree of fibrosis in heart tissues collected from congenital heart surgery patients with low pulse oxygen saturation and mice housed under chronic hypoxic and atmospheric pressure conditions was negatively correlated with pulse oxygen saturation. Moreover, TUG1 expression was positively correlated with the degree of fibrosis but negatively correlated with pulse oxygen saturation. Cardiac fibroblasts showed increased myofibroblast marker, collagen I and $\alpha$-SMA expression levels as the hypoxia time increased. TUG1 knockdown ameliorated the hypoxia-induced FMT. A bioinformatics analysis predicted that TUG1 had miR-29c binding sites in its 3'-UTR and miR-29c is a key regulator of cardiac fibrosis. The present study demonstrated that TUG1, along with miR-29c, may contribute to cardiac FMT activation and promote fibrosis in chronic hypoxia.
\end{abstract}

\section{Introduction}

Cyanotic congenital heart disease is one of the most common innate malformations in infants and children and is an important group of complex cardiac defects that is characterized by

Correspondence to: Professor Yingbin Xiao or Professor Zhao Jian, Department of Cardiovascular Surgery, Xinqiao Hospital, Army Medical University, 183 Xinqiao Street, Chongqing 400037, P.R. China

E-mail: xiaoyb@tmmu.edu.cn

E-mail: zjian918@yahoo.com

Key words: hypoxia, cardiac fibroblast, cardiac myofibroblast, TUG1, miR-29c various cardiac defects and decreased oxygenation. The influence of hypoxia on interstitial fibrosis has been reported by several studies in both the heart and other organs $(1,2)$. Cardiac fibrosis accompanied by heart failure occurs in patients with different heart disease etiologies. Specific and effective anti-fibrotic strategies and therapies are not available in the clinic. The main molecular mechanism of cardiac fibrosis is over-activated fibroblasts and excessive accumulation of extracellular matrix (ECM) (3). Therefore, further understanding of cardiac fibrosis in hypoxia might provide insights into strategies to help restore heart function in cyanotic heart disease and other hypoxia-related heart injuries.

Accumulating evidence demonstrates that noncoding RNAs (ncRNAs) are involved in physiological and pathological processes in the heart (4). ncRNAs shorter than 200 nucleotides are defined as short/small ncRNAs. One class of these is well known and are called microRNAs (miRNAs), which are small endogenous RNA molecules that mediate specific transcriptional repression or target mRNA degradation. The role of miRNAs in various heart diseases has already garnered considerable attention, for example, miR-1 and miR-133 function in cardiac development, miR-24 plays a role in cardiac angiogenesis, the miR-29s family plays a role in ECM (5-7). With a deeper knowledge of ncRNAs, long noncoding RNAs (lncRNAs) are gaining interest among researchers all over the world. LncRNAs are defined as transcripts of longer than 200 nucleotides from the genome. Numerous lncRNAs, such as MALAT1, NEAT1, NEAT2, HOTAIR, H19, and Taurine Upregulated Gene 1 (TUG1), participate in various types of cardiac development and diseases $(8,9)$. Currently, lncRNAs are mainly known to act as i) molecular signals; ii) 'sponges' or competing endogenous RNAs (ceRNAs); iii) guide lncRNAs; and iv) scaffold RNAs $(10,11)$. Of these, their function as sponges or ceRNAs to miRNAs have cast attention back onto miRNAs. The IncRNA CHRF has been reported to regulate cardiac hypertension by targeting miR-489 (11).

Previous studies have identified fibrosis in hypoxic cardiac disease, while most of the studies focus on the myocardial infarction model which includes both of ischemia and hypoxia (12). And there are evidences that miRNAs and lncRNAs mediate hypoxic fibrosis in other organs $(13,14)$. For example, the miR-214 promoted renal tubular epithelial cell mesenchymal transition and enhanced renal fibrosis induced by hypoxia (15). In addition, TET3 participated in liver fibrosis via modulating the expression of long non-coding RNA HIF1A-AS1 (16) and 
IncRNA ATB promoting pulmonary fibrosis by competitively binding miR-200c (17). Here, we report that chronic hypoxia promoted cardiac fibrosis in vitro and in vivo. Moreover, long noncoding RNA TUG1 inhibition could decrease hypoxia induced cardiac fibrosis through inhibiting cardiac fibroblast transformation to myofibroblasts, and these effects could be mediated by miR-29c.

\section{Materials and methods}

Human samples. The investigation conformed with the principles outlined in the Declaration of Helsinki. Informed consent was obtained from all of the subjects before participation. Human right ventricular outflow tracts (RVOTs) were obtained from a total of 19 patients who had cardiac operations for congenital heart diseases in our department. Seven patients with an $\mathrm{SpO}_{2}$ (pulse oxygen saturation) of less than $85 \%$ were assigned to the cyanotic group, seven patients whose $\mathrm{SpO}_{2}$ was above $95 \%$ were assigned to the acyanotic group, and the other 5 patients were assigned to the middle group. The clinical data of these patients is shown in Table I. Samples of human stenotic right outflow tracts were immediately preserved in $4 \%$ poly formaldehyde or liquid nitrogen. All procedures in our study were approved by the Human Ethical Committee of Xinqiao Hospital.

Animals and hypoxia treatment. All animal protocols were approved by the Institutional Animal Care and Use Committee of the Army Medical University. All of the experiments were performed on age-matched male mice. A hypoxia workstation (BAKER RUSKINN's InvivO2-1000) maintaining a $10 \% \mathrm{O}_{2}$ environment content at $24^{\circ} \mathrm{C}$ was used to raise the 8 -week-old mice for four weeks to mimic chronic hypoxia conditions (hypoxic group, $\mathrm{n}=10$ ). A group of age-, weight- and sex-matched C57 BL/6 mice were exposed to $21 \% \mathrm{O}_{2}$ condition for 4 weeks (normoxic group, n=10; Fig. 1). There were 10 mice in each group.

Cell culture and hypoxia treatment. Adult cardiac fibroblasts were isolated from 12-week-old mice. Briefly, 3-5 adult mouse hearts were obtained from freshly euthanized mice, cut into $1-\mathrm{mm}^{3}$ granules and digested with a mixture of $0.1 \%$ trypsin and $0.1 \%$ collagenase II in a $37^{\circ} \mathrm{C}$ water bath for $1 \mathrm{~h}$. Then, the digested products were filtered through a 200 -mesh screen filter to remove undigested cellular aggregates, non-cellular tissue components and debris and were centrifuged at 1,000 rpm for $5 \mathrm{~min}$. The cell pellet was resuspended in DMEM/F12 with 10\% FBS (Fetal Bovine Serum), and then the cells were plated and incubated in $95 \%$ air and $5 \% \mathrm{CO}_{2}$ at $37^{\circ} \mathrm{C}$ for $1 \mathrm{~h}$. The supernatant was discarded, and new culture medium was added. We cultured the cardiac fibroblasts in a $1 \% \mathrm{O}_{2}$ and 5\% $\mathrm{CO}_{2}$ cell incubator (Thermo Fisher Scientific, Inc., Waltham, MA, USA) for the hypoxia treatment for $0,6,12$ and $24 \mathrm{~h}$.

Transfection. Adult cardiac fibroblasts were cultured to $80 \%$ confluence in 6-well plates and transfected with three short hairpin RNAs (shRNAs) targeting TUG1 (sh-TUG1 1, sh-TUG1 2 and sh-TUG1 3) and the negative control shRNA were inserted into GV102 vectors (sh-NC), which were all synthesized by GeneChem Corporation (Shanghai, China).
The miR-29c mimics and the negative controls were purchased from Ribobio Corporation (Guangzhou, China). The miR-29 mimics, sh-TUG1s or their respective negative controls were then transfected into cells. The predicted binding sites of TUG1 and miR-29c and their corresponding mutations were respectively recombined into a vector containing the luciferase gene. Each of these vectors or negative controls were cotransfected with the miR-29c mimic or its negative control into HEK293 cells for $48 \mathrm{~h}$ using the Fugene ${ }^{\circledR} 6$ Transfection Reagent (Promega Corporation, Madison, WI, USA) according to the manufacturer's protocol. Transfected cells were cultured for an additional $24 \mathrm{~h}$ prior to hypoxia treatment.

Cell proliferation assay. We performed a Cell Counting Kit-8 (CCK8) assay (Beyotime, China) to analyze cell proliferation. The CCK-8 reagent was added to each well in their own condition with or without hypoxia culture, and the cells were incubated for $2 \mathrm{~h}$. The optical density (OD) of each well was recorded at $450 \mathrm{~nm}$ on a microplate reader (Thermo Fisher Scientific, Inc.).

Masson's trichrome staining. Masson's trichrome was used to stain all forms of collagen in the heart. All of the tissues maintained in $4 \%$ poly formaldehyde were embedded in paraffin and cut into 5- $\mu \mathrm{m}$ sections. After dewaxing the sections, stained the sections with Mayer-s Hematoxylin, Ponceau S, $1 \%$ phosphomolybdic acid aqueous solution and aniline blue (Beijing Solarbio Science \& Technology Co., Ltd., Beijing, China) successively, and dehydrated in 95\% ethyl alcohol (Chuandong Chemical Industry, Chongqing, China) several times, followed by anhydrous alcohol (Chuandong Chemical Industry). Finally, the samples were hyalinized with dimethylbenzene (Beijing Solarbio Science \& Technology Co., Ltd.) and sealed with neutral balsam (ZSGB-Bio, Beijing, China). The stain for the collagen fibers was blue, the cytoplasm, muscle fibers and red blood cells were red, and the nuclei were black.

The staining sections were performed on a Leica inverted microscope. The images were captured 5 different vision from one section and we used average fibrotic area ratio of one section as the average fibrotic level from one sample The fibrotic area ratio was quantified by calculating the percentage of collagen staining (blue staining) area on the whole section area (all the staining tissue areas) using Image-pro Plus v.6.0.

Immunofluorescence staining. The frozen tissues were embedded in OCT and sectioned to $6 \mu \mathrm{m}$ thickness. After being fixed with $4 \%$ paraformaldehyde, the sections were blocked with normal goat serum for $1 \mathrm{~h}$ and then incubated with primary antibodies overnight at $4{ }^{\circ} \mathrm{C}$. And the sections were incubated with the corresponding secondary antibodies conjugated to Alexa Fluor 488 or 647 (Beyotime Institute of Biotechnology, Haimen, China). The following primary antibodies were used: Anti-Vimentin (1:100; BosterBio Co., Ltd., Wuhan, China) and anti- $\alpha$-SMA (1:200, Sigma-Aldrich; Merck KGaA, Darmstadt, Germany). Immunofluorescence was performed on a Leica confocal microscopy system.

RNA extraction and quantitative real-time PCR ( $q R T-P C R)$. Total RNA was extracted from human tissue, mouse tissue and cells using TRIzol (Invitrogen; Thermo Fisher Scientific, Inc.). A total of $1 \mathrm{ml}$ TRIzol was added to $100 \mathrm{mg}$ of tissue or to 
Table I. Clinical data of patients.

\begin{tabular}{lccc}
\hline Clinical data & Cyanotic group & Middle group & Acyanotic group \\
\hline Age at operation (years) & $11.05 \pm 13.82$ & $17.65 \pm 18.39$ & $5.70 \pm 9.91$ \\
Sex (Male/Female) & $5 / 2$ & $3 / 2$ & $5 / 1$ \\
Oxygen saturation (\%) & $75.00 \pm 8.02^{\mathrm{a}}$ & $89.20 \pm 1.92^{\mathrm{a}}$ & $97.14 \pm 1.46^{\mathrm{a}}$ \\
LVEF (\%) & $67.26 \pm 5.88$ & $70.58 \pm 2.64$ & $71.69 \pm 5.81$ \\
LVFS (\%) & $34.79 \pm 4.80$ & $39.44 \pm 2.49$ & $40.10 \pm 4.71$ \\
Pathology & & & 1 \\
TOF & 4 & & 2 \\
TOF \& PA corrective postoperation & 3 & 3 & 5 \\
VSD \& ROVS & & 1 & 5 \\
VSD \& PAH & & &
\end{tabular}

LVEF, left ventricular ejection fraction; LVFS, left ventricular shortening fraction; TOF, tetralogy of Fallot; PA, pulmonary atresia; VSD, ventricular septal defect; ROVS, right ventricular outflow tract obstruction; $\mathrm{PAH}$, pulmonary arterial hypertension. ${ }^{\mathrm{a}} \mathrm{P}<0.05$ compared with each of the other groups.

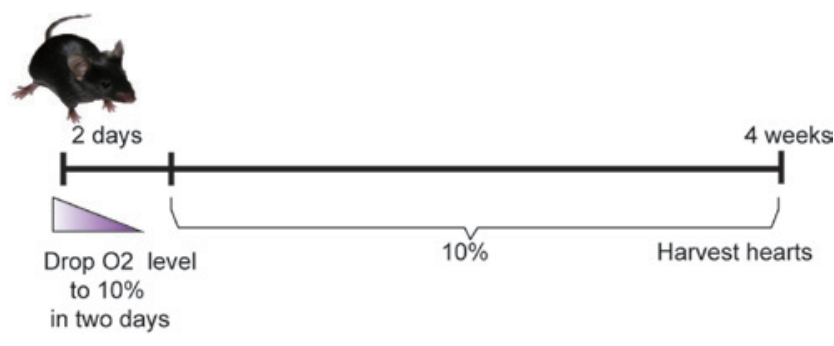

Figure 1. Schematic diagram of mice hypoxia model.

$10^{6}$ cells, and the subsequent steps were performed according to the manufacturer's instructions. RNA concentration and purity were estimated using a NanoDrop 1000 (Thermo Fisher Scientific, Inc.). Reverse transcription was carried out using the RT PrimeScript ${ }^{\mathrm{TM}}$ RT reagent kit (Perfect Real Time; Takara Bio, Inc., Otsu, Japan) according to the manufacturer's instructions. Amplification of the cDNA products was performed with SYBR ${ }^{\circledR}$ Premix Ex Taq ${ }^{\mathrm{TM}}$ II (Perfect Real Time; Takara Bio, Inc.) on an Applied Biosystems 7500 Fast Real-Time PCR System (Thermo Fisher Scientific, Waltham, MA, USA). The amplification kit used U6 snRNA as the internal control for the microRNAs and used $\beta$-actin (ACTB) as the internal control for the lncRNAs. The primers for the miR-29 families and U6 detection were purchased from Guangzhou RiboBio Co., Ltd, (Guangzhou, China), and the primers for ACTB and TUG1s were synthesized by Shengong Biotech Co., Ltd., (Shanghai, China) and are shown in Table II.

Protein extraction and western blotting. Total protein was extracted from tissues and cell cultures using Cell Extraction Buffer (Thermo Fisher Scientific, Inc.) and the Mini Protease Inhibitor Cocktail (Roche Diagnostics, Basel, Switzerland), and proteins were separated via SDS-PAGE. The gels were wet transferred to PVDF membranes (Roche Diagnostics), incubated overnight with primary antibodies, and then incubated with secondary antibodies. Immunoreactivity was revealed by chemiluminescence using a western blot imaging system (GE Healthcare, Chicago, IL, USA), and the gray value
Table II. Primers of human long non-coding RNAs.

\begin{tabular}{ll}
\hline Gene & \multicolumn{1}{c}{ Primers } \\
\hline Human-ACTB & F 5'-CATGTACGTTGCTATCCAGGC-3' \\
& R 5'-CTCCTTAATGTCACGCACGAT-3' \\
Human-TUG1 & F 5'-GCACCAGTACAAGCAGCAGA-3' \\
& R 5'-AATGGCACCCAGTGTAAAGC-3' \\
Mouse-ACTB & F 5'-GGCTGTATTCCCCTCCATCG-3' \\
& R5'-CCAGTTGGTAACAATGCCATGT-3' \\
Mouse-TUG1 & R 5'-CTGCCACAACCATCTTCCTT-3' \\
& F 5'-TGCCATCCAGGTCAAACAT-3'
\end{tabular}

F, forward; R, reverse.

was analyzed by ImageJ $1.50 \mathrm{i}$. $\beta$-actin was used as the internal reference. The normoxic group was used as the control for the protein level analysis in the mouse tissue protein, and the $0-h$ hypoxia cultured cells, untransfected and normoxic cultured cells served as controls for their own groups. All of the samples were run at least in triplicate. The antibodies utilized were $\alpha$-SMA $(1: 1,000)$, collagen I $(1: 1,000)$ and beta-actin (internal reference; 1:1,000; Boster Co., Ltd).

Dual-luciferase reporter gene assay. The predicted targeted sequence wide type (WT) of TUG1 and a mutated (MUT) sequence of TUG1 with mutations at the predicted binding site of miR-29c were inserted into the GV272 control vector in the XbaI site, which was purchased from GeneChem, Inc., (Shanghai, China). For the dual-luciferase assay, HEK293 cells were transfected with the WT vector and the miR-29c mimics or the MUT vector and the miR-29c mimics. Following transfection, the activities of Firefly luciferase and Renilla luciferase were measured with a dual-luciferase reporter assay kit (Promega E1910; Promega Corporation) on a luminometer (i3x; Molecular Devices, LLC, Sunnyvale, CA, USA) according to the manufacturer's instructions. 

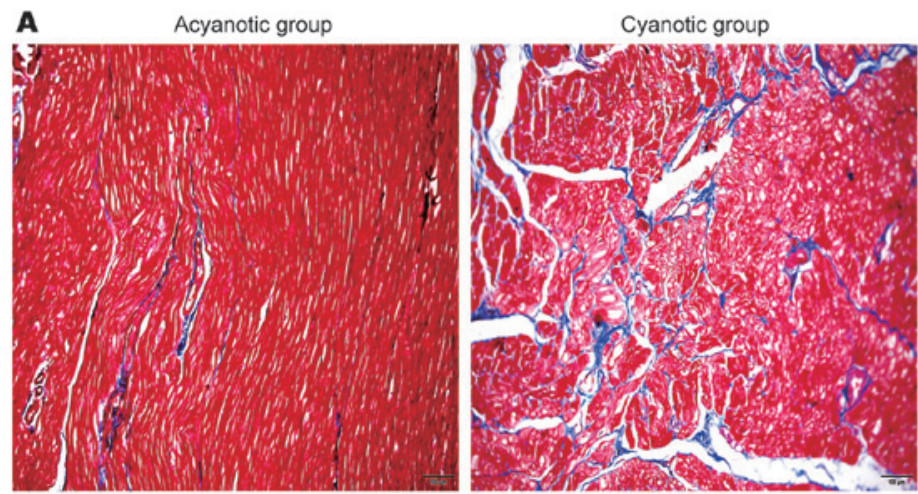

D
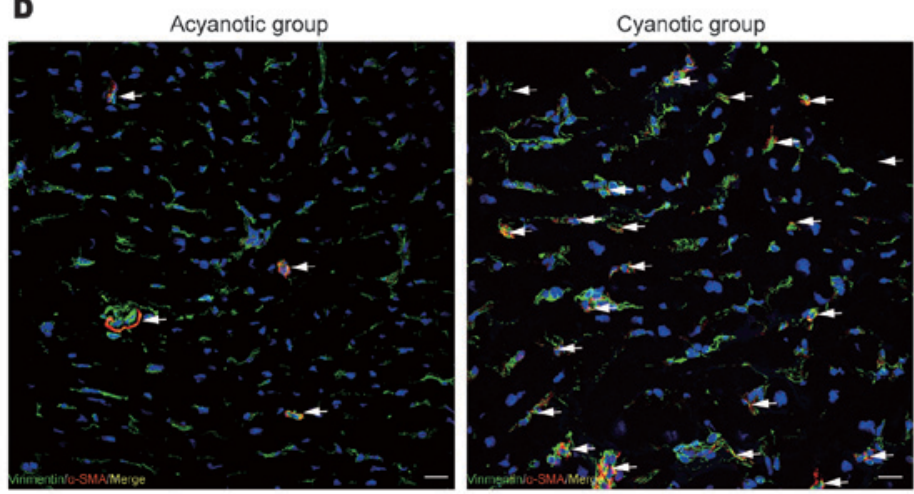

$\mathbf{F}$

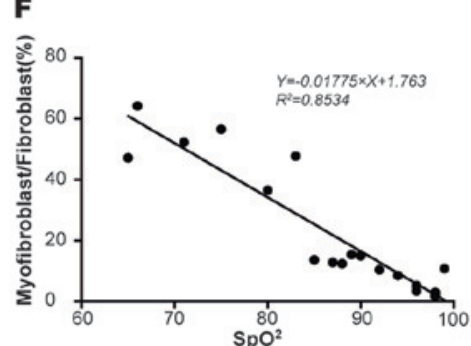

G

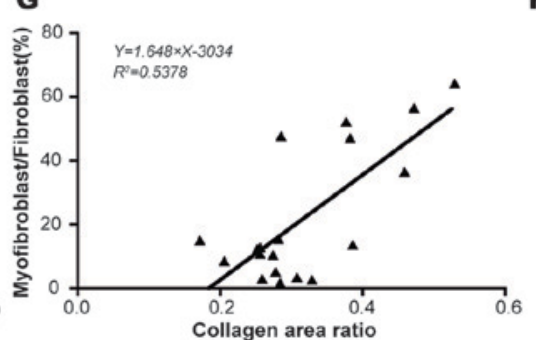

B

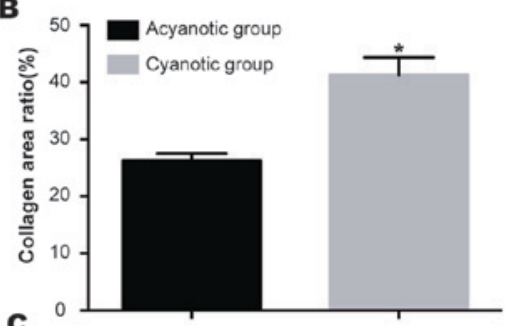

C
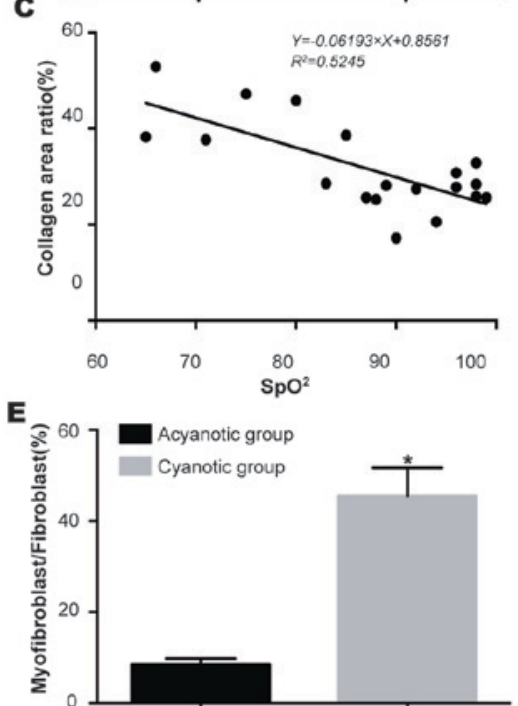

H

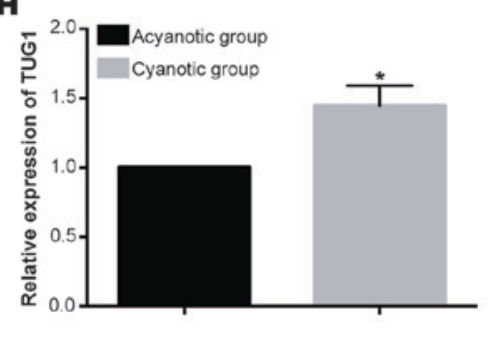

Figure 2. Heavier fibrosis and higher TUG1 expression in cyanotic group than acyanotic group. (A) Histological sections of myocardial tissue of the patients stained with Masson trichrome (magnification, $\mathrm{x} 100$; bar indicates $50 \mu \mathrm{m}$ ). (B) The statistic results of Masson trichrome ("P<0.05 vs. acyanotic group). (C) The correlation analysis between collagen area ratio and $\mathrm{SpO}_{2}$. (D) Co-immunostaining of myocardial tissue sections marked with vimentin (Green) and $\alpha$-SMA (Red) (magnification, $\mathrm{x} 400$; bar indicates $12.5 \mu \mathrm{m}$ ), and the co-localization (Yellow) of these two makers means the myofibroblast which were signed with white arrows. (E) The statistic results of laser confocal microscopy (" $\mathrm{P}<0.05$ vs. acyanotic group). (F) The correlation analysis between myofibroblast ratio and showed $\mathrm{SpO}_{2}$. (G) The correlation analysis between collagen area ratio and myofibroblast ratio. (H) The statistic results of TUG1 relative expression in clinical samples ( $\mathrm{P}<0.05$ vs. acyanotic group). TUG1, Taurine Upregulated Gene 1.

Statistical analysis. Data are presented as the mean \pm SEM. Statistical significance was evaluated using unpaired Student's T-tests with Welch's correction for comparing two groups. For comparing three or more groups, the means were compared using one-way analysis of variance accounting for differences in variance among the groups. Post hoc pairwise comparisons were performed using either the LSD or Tukey-Kramer method. Data were analyzed using GraphPad Prism 6 (GraphPad Software, Inc., La Jolla, CA, USA) or SPSS v.22.0 (IBM Corp., Armonk, NY, USA) statistical software.

\section{Results}

Cardiac fibrosis deteriorated and TUG1 was upregulated with decreasing oxygen saturation in congenital heart disease patients. To evaluate whether the degree of fibrosis was correlated with oxygen saturation, we collected (RVOT) muscle from
19 patients ( 7 cyanotic, 7 acyanotic and 5 middle). The clinical characteristics are summarized in Table I. We divided the patients into three groups according to the $\mathrm{SpO}_{2}$, with the cyanotic group being below $85 \%$, the acyanotic group being above $95 \%$ and the rest of the patients was the middle group. Age, sex and body weight were all matched between the cyanotic and acyanotic groups. We evaluated cardiac fibrosis via Masson's trichrome labeling (Fig. 2A). An analysis of the Masson's trichrome-stained tissue sections from the cyanotic patients showed a significant deterioration of fibrosis area ratio (collagen area ratio) compared with that of the acyanotic patients (Fig. 2B). We also analyzed the $\mathrm{SpO}_{2}$ and degree of fibrosis in the sections of all 19 patients, and found that the degree of fibrosis was negatively correlated with the oxygen saturation (Fig. 2C). Given that the fibroblast-to-myofibroblast transition (FMT) is a critical component of cardiac fibrosis, we examined the myofibroblast ratio of these sections via confocal microscopy (Fig. 2D). The myofibroblast/fibroblast 

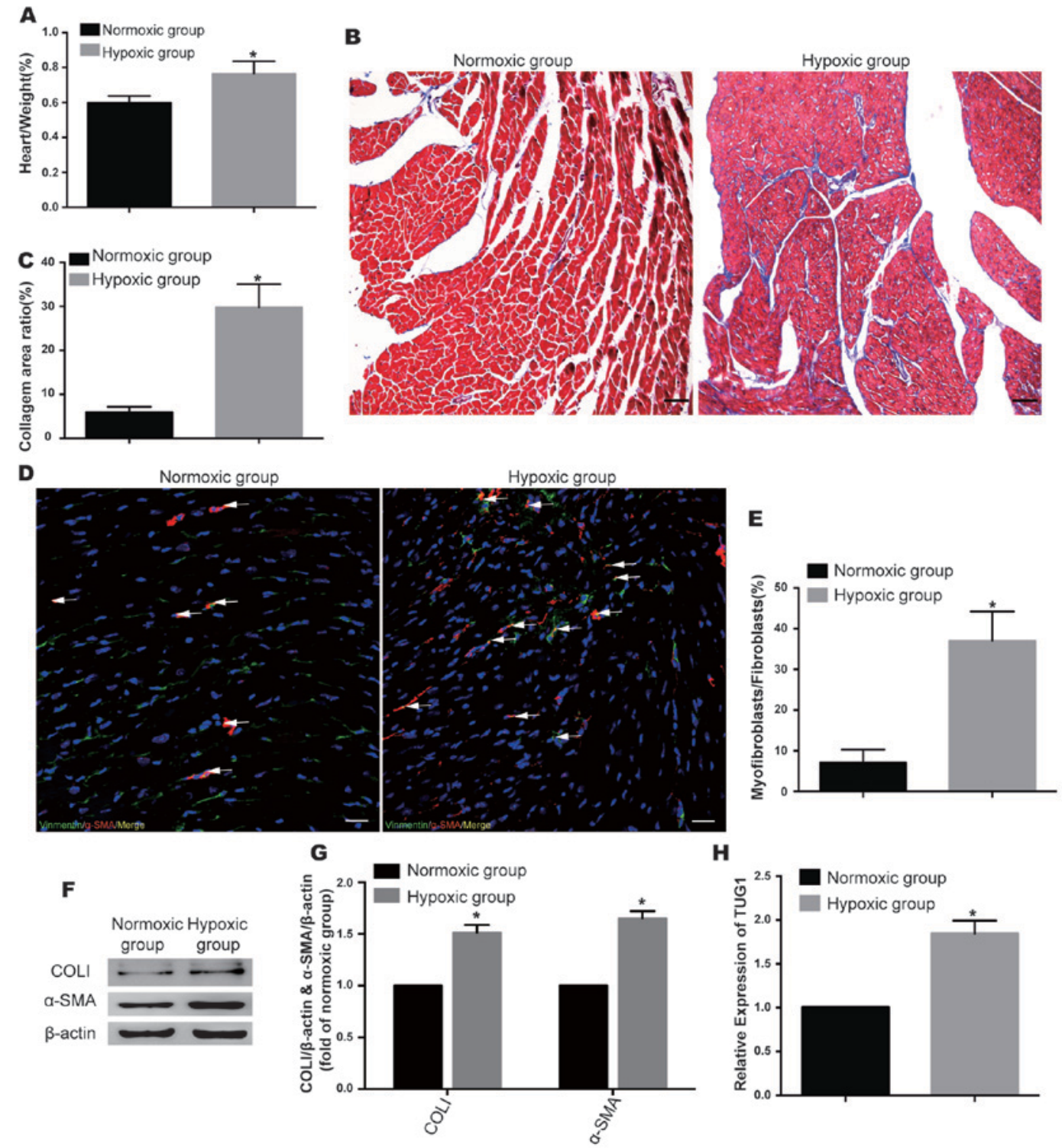

Figure 3. Hypoxia induced cardiac fibrosis and TUG1 level increased in mice model. (A) Ratio of HW to BW. (B) Masson trichrome stain of myocardial tissue sections (magnification, x100; bar indicates $50 \mu \mathrm{m}$ ). (C) The statistic results of Masson trichrome (*P<0.05 vs. normoxic group). (D) Co-immunostaining of Vimentin (Green) and $\alpha$-SMA (Red) in myocardial tissue (magnification, $\mathrm{x} 400$; bar indicates $12.5 \mu \mathrm{m}$ ). (E) The statistic results of Masson trichrome and co-immunostaining ( ${ }^{*} \mathrm{P}<0.05$ vs. normoxic group). (F) Western blots of the myocardial tissue from hypoxic and normoxic group (all samples were derived at the same time and processed in parallel). (G) The band analysis of western blot ("P<0.05 vs. normoxic group). (H) The statistic results of TUG1 relative expression in mice ( $\mathrm{P}<0.05$ vs. normoxic group). TUG1, Taurine Upregulated Gene 1; HW, heart weight; BW, body weight.

ratio was consistent with the Masson's trichrome staining, with the cyanotic group showing a higher myofibroblast ratio than the acyanotic group (Fig. 2E). The ratio was negatively correlated with the oxygen saturation in the tissue samples of all 19 patients (Fig. 2F), while it was positive correlated to the collagen area (Fig. 2G). Recently, IncRNAs were reported to participate in the physiological and pathological progression of the heart (18), and we found that TUG1 lncRNA expression was increased in the cyanotic patients compared with the acyanotic patients (Fig. $2 \mathrm{H}$ ). These data indicated that cardiac fibrosis was enhanced in heart tissues with lower oxygen saturation and that TUG1 expression was coincident with the level of cardiac fibrosis.

Cardiac fibrosis was exacerbated and TUG1 expression was increased in chronic hypoxic mice. Compared with the normoxic group, the hypoxic mice weighed less but had a greater heart/body weight ratio (Fig. 3A). We measured the degree of cardiac fibrosis by Masson's trichrome staining. The Masson's trichrome staining showed that the fibrotic area ratio (collagen area ratio) were significantly greater in the hypoxic group than in the normoxic group (Fig. 3B and C). We also evaluated the myofibroblast ratio via confocal microscopy, and the myofibroblast ratio of the hypoxic group was significantly greater than that in the normoxic group (Fig. 3D and E). In addition, western blotting revealed that the cardiac tissue had higher relative expression of collagen I (COLI) and $\alpha$-SMA than in the normoxic group (Fig. 3F and G), and one of these (COLI) is a major component of fibrotic lesions and the other $(\alpha-$ SMA) is a marker of the differentiation of fibroblasts to myofibroblasts. Meanwhile, the level of TUG1 was increased $(1.84 \pm 0.48$-fold $)$ in the hypoxic group compared with that in the normoxic group. These results indicated that hypoxia is 
A

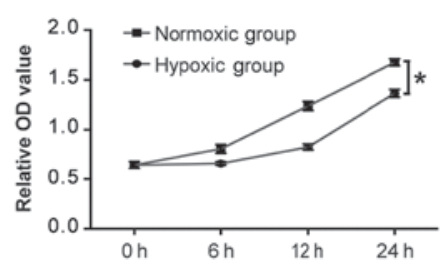

B
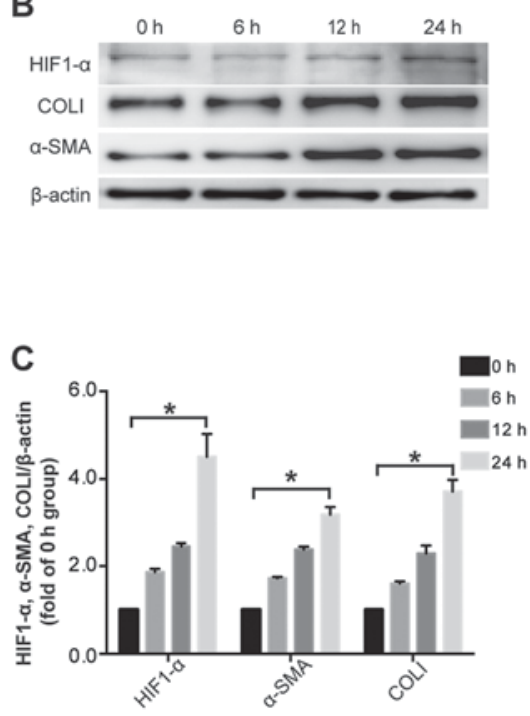

D
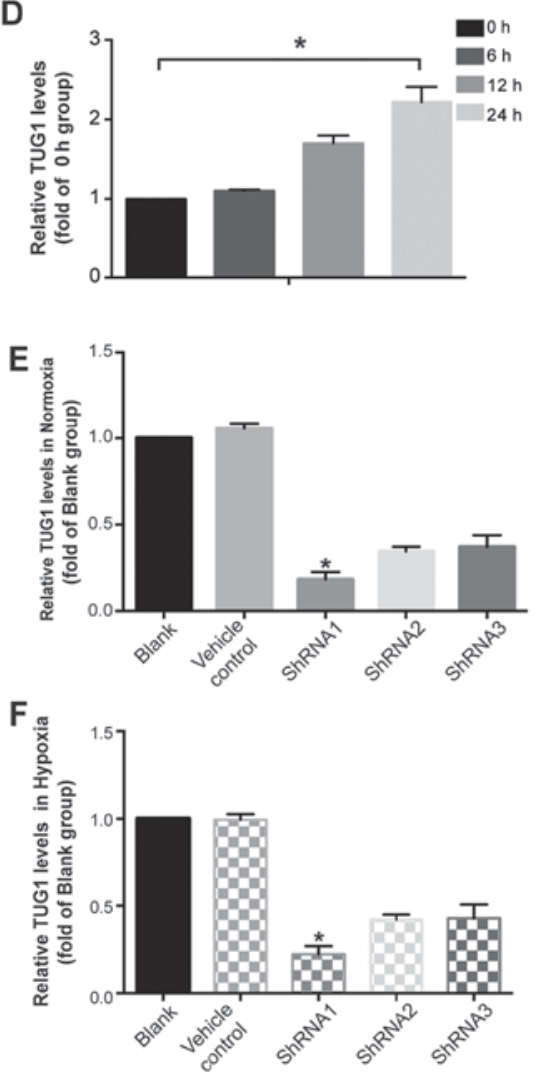

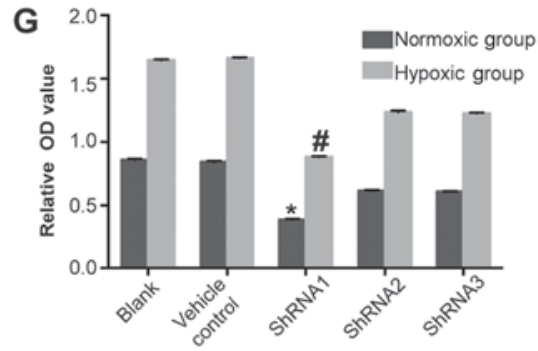

H

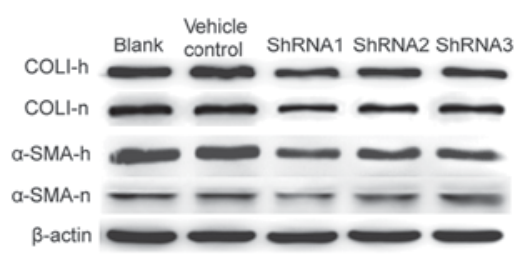

I

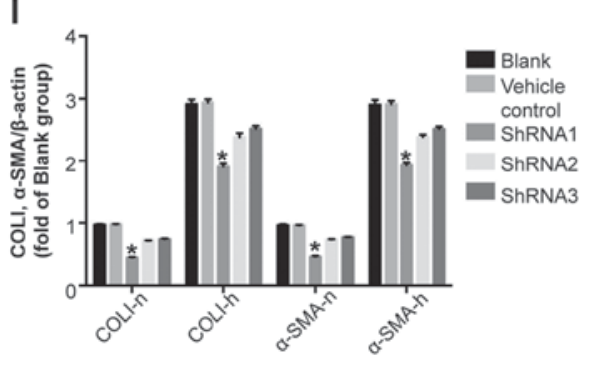

Figure 4. TUG1 knockdown alleviated the FMT induced by hypoxia. (A) The OD value of CCK-8 kit showed the proliferation of cardiac fibroblasts increased with the hypoxic time ( ${ }^{*} \mathrm{P}<0.05$ vs. normoxic group). (B) The expression of HIF-1 $\alpha$, COLI and $\alpha$-SMA in fibroblasts at different hypoxia time point (All samples were derived at the same time and processed in parallel). (C) The gray value of western blot of HIF-1 $\alpha$, COLI and $\alpha-S M A$ ("P<0.05 vs. 0 h group). (D) The relative expression of TUG1 at different hypoxic time point ( $\mathrm{P}<0.05$ vs. $0 \mathrm{~h}$ group). (E and F) The relative expression of TUG1 after transfection with three TUG1 shRNA (shRNA1, shRNA2 and shRNA3) and its negative control in normoxic condition and hypoxic condition ("P<0.05 vs. each group). (G) The OD value of CCK-8 kit showed the proliferation of cardiac fibroblasts transfected with TUG1 shRNA (shRNA1, shRNA2 and shRNA3) and its negative control in normoxic and hypoxic condition ( $\mathrm{P}<0.05$ vs. each group in normoxia, ${ }^{\#} \mathrm{P}<0.05$ vs. each group in hypoxia). (H) The expression of COLI and $\alpha$-SMA in fibroblasts transfected with TUG1 shRNA (shRNA1, shRNA2 and shRNA3) and its negative control in normoxic(-n) and hypoxic(-h) condition (All samples were derived at the same time and processed in parallel). (I) The gray value of western blot of COLI and $\alpha-$ SMA ( ${ }^{*}<0.05$ vs. each group). TUG1, Taurine Upregulated Gene 1; FMT, fibroblast-myofibroblast transformation; COLI, collagen I.

strongly correlated with FMT and fibrosis, and TUG1 might play a role in this process.

TUG1 knockdown alleviated the FMT induced by hypoxia. To further confirm the role of TUG1 in cardiac FMT induced by hypoxia, we detected the cardiac fibroblast proliferation and the expression of COLI, $\alpha$-SMA and TUG1 in the cardiac fibroblasts under normoxic and hypoxic conditions. The CCK-8 results showed that cardiac fibroblast proliferation increased with the hypoxic time (Fig. 4A). The COLI and $\alpha$-SMA levels increased with the hypoxic time, which was coincidence with the HIF-1 $\alpha$ expression (Fig. 4B and C). Furthermore, TUG1 expression was also increased with the hypoxic time (Fig. 4D). We next knocked down the expression of TUG1 by transfecting cardiac fibroblasts with vectors containing the TUG1 shRNAs (shRNA1, shRNA2 and shRNA3) or the vehicle control (empty vector). All three TUG1 shRNAs decreased TUG1 expression, with the greatest decrease observed with shRNA1 (Fig. 4E). After the transfected cells were cultured under hypoxic conditions for $24 \mathrm{~h}$, the levels of TUG1 were increased. Although the expression of TUG1 increased under hypoxia, the levels in the knockdown groups (using the three TUG1 shRNAs) still remained lower than in those in the blank and negative groups
(Fig. 4F). We also found that proliferation was significantly decreased in the TUG1 shRNA-transfected cells, especially those transfected with shRNA1 (Fig. 4G). Cell proliferation was still increased under hypoxia though the levels were lower in the three knockdown groups. The COLI and $\alpha$-SMA levels were also decreased in the shRNAs-transfected cells compared with those in the blank and vehicle control cells (Fig. $4 \mathrm{H}$ and I). Besides, COLI and $\alpha$-SMA levels were all increased after hypoxia but the levels were lower in the three knockdown groups, which was consistent with the TUG1 level (Fig. 4H and I). Therefore, we concluded that TUG1 facilitated cardiac FMT in hypoxia.

TUG1 regulated the cardiac FMT induced by hypoxia via $\mathrm{miR}-29 c$. Some researchers report that miR-29c shows reduced expression in TOF patients according to microarray analysis (19) and is related to hypoxia. Increasing evidence indicates that the miR-29s family plays an important role in cardiac fibrosis $(20,21)$. We found that TUG1 expression was correlated with the miR-29s family in human breast, kidney and lung cancer tissues from the starBase V2.0 database, and the predicted target sites of miR-29c in human TUG1 are shown in Fig. 5A. Considering the conservation of lncRNAs 
A

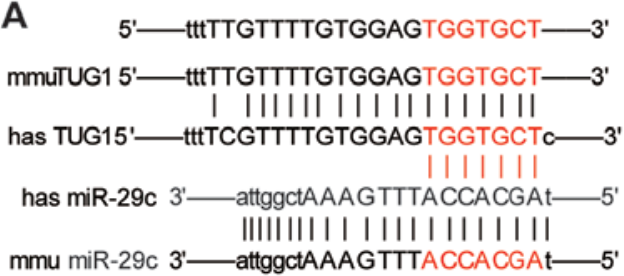

B

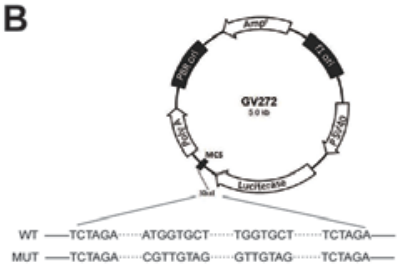

C
D

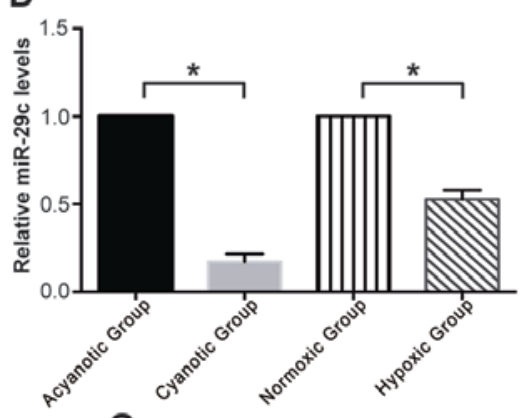

E

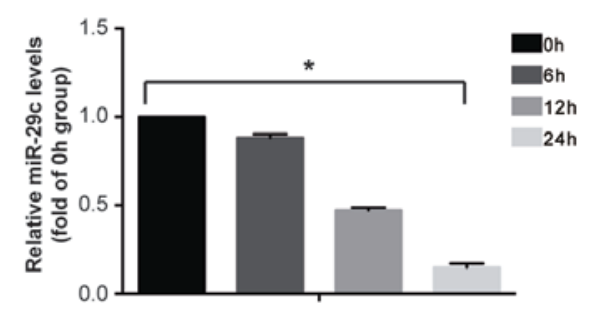

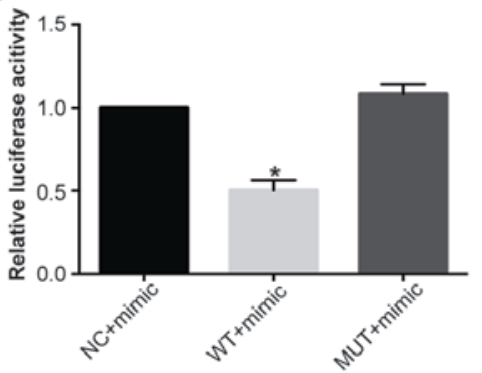
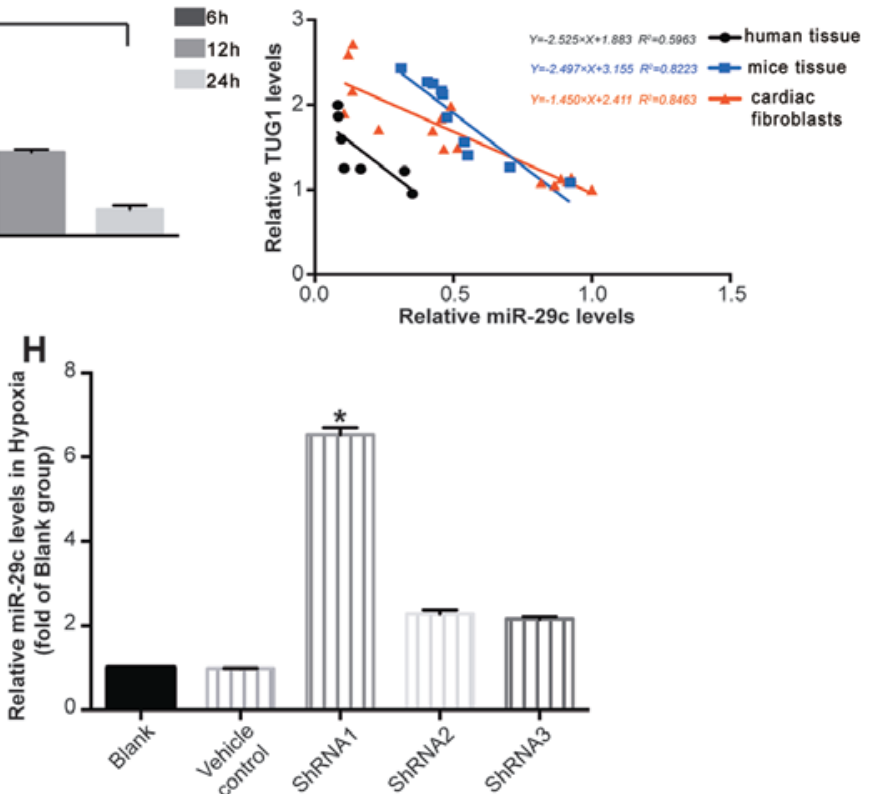

Figure 5. TUG1 via miR-29c regulated the cardiac FMT. (A) The predicted binding sites of TUG1 and miR-29c were marked in red, and the comparison of the sequence of TUG1 and miR-29c of human and mice. (B) The vector GV272 were recombined with predicted binding sites (WT or MUT) of TUG1 and miR-29c with XbaI site. (C) The dual-luciferase result showed the WT+mimic group had lower relative luciferase value (*P<0.05 vs. each group) (C) The relative expression levels of miR-29c of human ( ${ }^{*} \mathrm{P}<0.05$ vs. acyanotic group) and mice tissues ( ${ }^{*} \mathrm{P}<0.05$ vs. normoxic group). (D) The relative expression of miR-29c of cardiac fibroblasts at different hypoxic time ( ${ }^{*} \mathrm{P}<0.05$ vs. 0 h group). (E) The relative expression of miR-29c of cardiac fibroblasts at different hypoxic time ( $\mathrm{P}<0.05$ vs. $0 \mathrm{~h}$ group). (F) The correlation analysis between miR-29c and TUG1 in human tissue, mice tissue and cardiac fibroblasts in hypoxia. (G and H) The relative expression of miR-29c after transfection with three TUG1 shRNA (shRNA1, shRNA2 and shRNA3) and its negative control in normoxic condition $\left({ }^{*} \mathrm{P}<0.05\right.$ vs. each group in normoxia) and hypoxic condition ( ${ }^{*} \mathrm{P}<0.05$ vs. each group in hypoxia). TUG1, Taurine Upregulated Gene 1; FMT, fibroblast-myofibroblast transformation; WT, wild type.

and microRNAs, we blasted the TUG1 and miR-29c target sites in the mice genome and found they were conserved (Fig. 5A). The information of recombined vectors showed in the Fig. 5B. The dual-luciferase reporter system results showed that the relative luciferase expression of the WT group with miR-29c mimics was significantly lower than those of the negative control group with miR-29c mimics and the MUT group with miR-29c mimics (Fig. 5C), confirming the predicted binding sites of TUG1 and miR-29c.

To further examine whether TUG1 participated in the cardiac FMT induced by hypoxia via miR-29c, we examined the miR-29c levels of the clinical samples and hypoxic mice. We found that the expression of miR-29c was lower in the cyanotic group and hypoxic group, compared with the acyanotic and normoxic group, respectively (Fig. 5D). The expression of miR-29c in cardiac fibroblasts appeared to decrease with the hypoxic time (Fig. 5E). All the human and mice tissues and the cell data indicated that the expression of TUG1 and miR-29c were negatively correlated (Fig. 5F). We then examined the miR-29c level after TUG1 knockdown.
MiR-29c expression was significantly increased in the cardiac fibroblasts transfected with TUG1 shRNAs, especially the cells transfected with shRNA1, whereas miR-29c expression decreased slightly with hypoxia (Fig. 5G and H). COLI is a target gene of miR-29c, and $\alpha$-SMA is also regulated by miR-29c in cardiac fibrosis (22). In conclusion, TUG1 might promote the cardiac FMT in hypoxia via miR-29c.

miR-29c overexpression ameliorates hypoxia-induced FMT. To further confirm the function of miR-29c in cardiac fibroblasts, we overexpressed miR-29c in adult mouse cardiac fibroblasts by treating them with an miR-29c sequence mimic and its negative control (Fig. 6A). Using the CCK8 assay, we found that the hypoxia group had higher OD values, whereas the mimic-treated cardiac fibroblasts had lower OD values in both of their own groups. Hypoxia stimulated the proliferation of cardiac fibroblasts, which was restrained to some extent by the overexpression of miR-29c (Fig. 6B). Confocal imaging revealed that $\alpha$-SMA expression was reduced in the normoxic group compared with that in the hypoxic group, and the mimic 

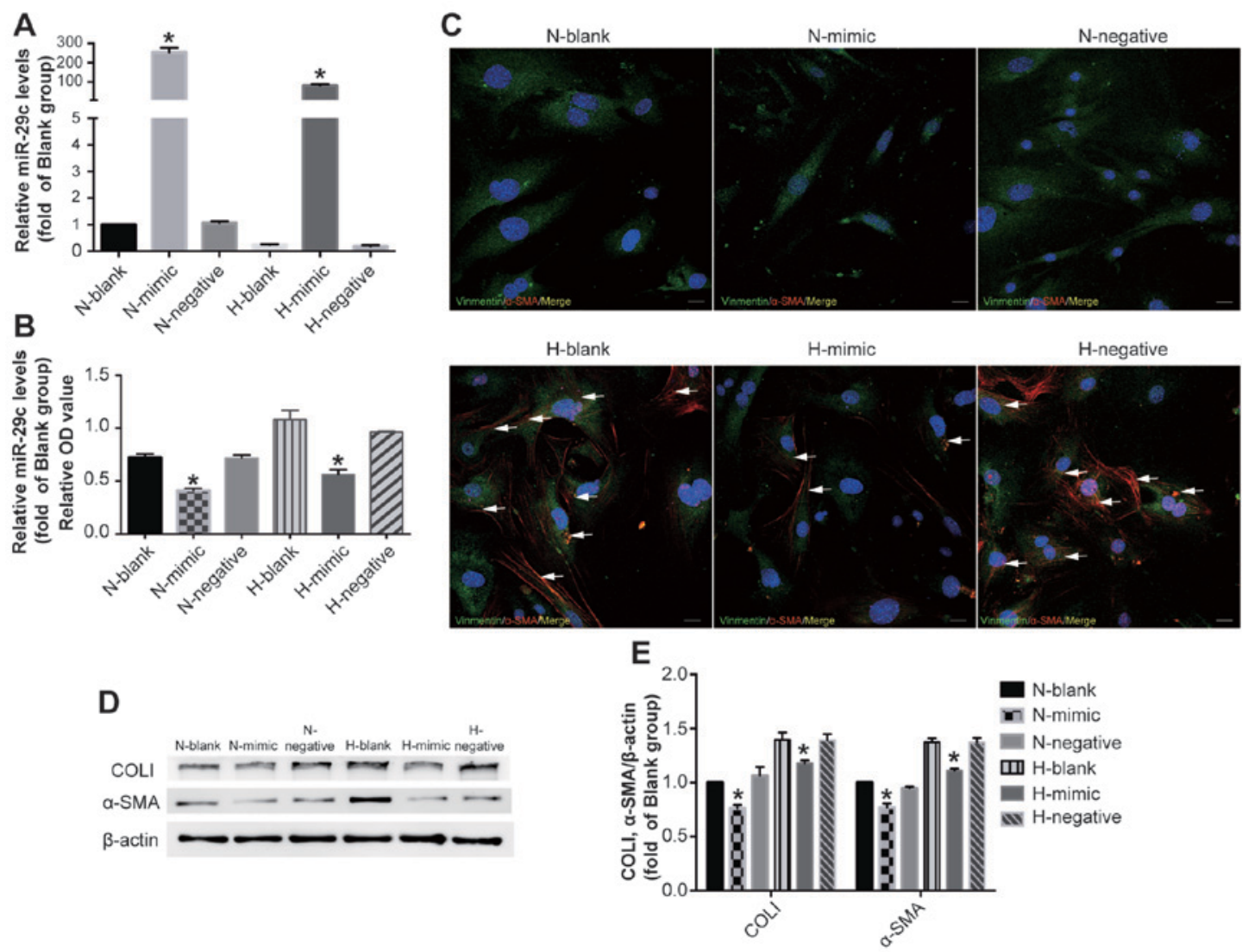

Figure 6. miR-29c regulated the cardiac FMT. (A) The relative expression of miR-29c in control, miR-29c mimic, and negative-control in both normoxic ( $\mathrm{P}<0.05$ vs. each group in normoxia) and hypoxic condition (" $\mathrm{P}<0.05$ vs. each group in hypoxia). (B) The CCK8 result of fibroblast in control, miR-29c mimic, and negative-control in both normoxic (" $\mathrm{P}<0.05$ vs. each group in normoxia) and hypoxic condition (" $\mathrm{P}<0.05$ vs. each group in hypoxia). (C) The co-immunostaining of $\alpha$-SMA and Vimentin in fibroblast in control, miR-29c mimic, and negtive-control in both normoxic and hypoxic condition (magnification, $\mathrm{x} 400$; bar indicates $12.5 \mu \mathrm{m}$ ). (D) Western blot of COLI and $\alpha$-SMA in control, miR-29c mimic, and negtive-control in both normoxic and hypoxic condition. (E) The gray value of western blot of COLI and $\alpha$-SMA ("P $<0.05$ vs. each group in hypoxia or in normoxia). FMT, fibroblast-myofibroblast transformation; $\mathrm{N}-$, normoxic condition; $\mathrm{H}-$, hypoxic condition.

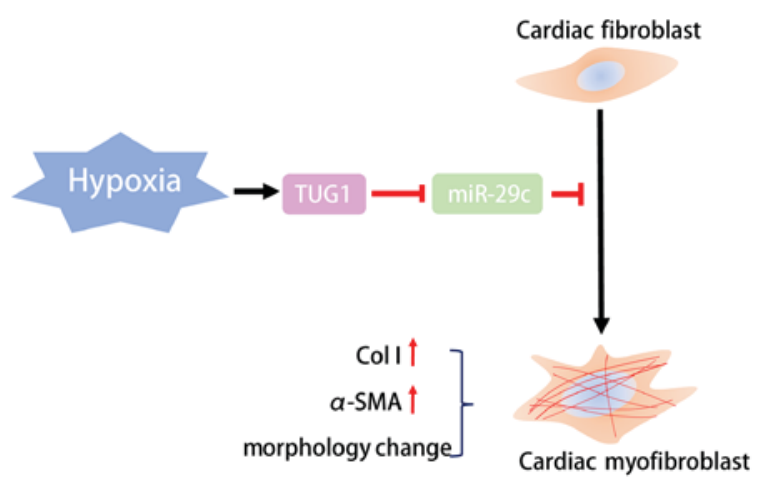

Figure 7. Schematic of the mechanism of TUG1 functioning as an miR-29c 'sponge'.

group under hypoxic conditions had lower $\alpha$-SMA expression than the control and negative control groups (Fig. 6C). In addition, western blotting indicated that the hypoxic group had greater $\alpha$-SMA and collagen I levels than the normoxic group, whereas the mimic-treated groups had lower $\alpha$-SMA and collagen I levels under both normoxic and hypoxic conditions (Fig. 6D and E). These results suggested that overexpressing miR-29c alleviates the elevated $\alpha$-SMA and collagen I levels induced by hypoxia, that is the overexpression of miR-29c indeed ameliorated activation of FMT induced by hypoxia. And higher level of TUG1 induced by hypoxia might restrain inhibiting effect of miR-29c on cardiac FMT. Collectively, we conclude the lncRNA TUG1 might function as the sponge of miR-29c to promote FMT in hypoxia (Fig. 7).

\section{Discussion}

Chronic hypoxia impairs cardiac function in a complex pattern, including not only the subsequent injury induced by pulmonary hypertension but also that induced by cardiac fibrosis. We confirmed the more cardiac fibrosis in chronic hypoxia both in the samples of chronic hypoxic patients and mice model. Nevertheless, the mechanism of fibrosis were complex and precisely regulated, which pattern of the cardiac fibrosis induced by hypoxia needs further exploration. Of the multiple key fibrogenic pathways that activate fibrosis progression, fibroblast proliferation, activation and transition to myofibroblasts is a major mechanisms of cardiac fibrosis. Although several reports have shown increased fibroblast proliferation in the hypoxic heart (23) and enhanced FMT under hypoxia condition in other organs $(24,25)$, most studies were performed on the ischemia disease or ischemic animal model. The present study confirmed FMT induced by normobaric hypoxia both in vitro and in vivo, 
which could reflect the pure influence of hypoxia on cardiac fibroblast better.

Accumulating evidence indicates that many lncRNAs participate in the physiological and pathological processes of heart $(8,26,27)$. Since being first identified in the murine retina (28), TUG1 has gained increasing attention for its role in multiple biological processes. TUG1 is a $6.7-\mathrm{kb}-$ long untranslated RNA molecule that participates in various carcinomas, contributing to tumor growth and development and playing some role in organ fibrosis $(29,30)$. Recently, TUG1 was reported to be upregulated in different hypoxic organs (31). The present study showed the higher TUG1 level in hypoxia samples and adult mouse cardiac fibroblasts in hypoxic culture, and inhibiting TUG1 ameliorated the FMT, which indicating TUG1 plays a role in the hypoxic FMT.

The precise molecular mechanisms underlying lncRNA function still remain thoroughly under investigation. Similarly, the pattern of TUG1 functioning encompassed from interacting with proteins to binding RNAs. He et al (32), reported TUG1 facilitated osteogenic differentiation of periodontal ligament stem cells via interacting with Lin28A. Zhang et al (33) presented TUG1 was required to target EZH2 occupancy and epigenetically modulate the expression of p15, p16, p21, p27 and p57, further regulated the cell proliferation. Yu et al (34) reported TUG1 sponged miR-204-5p to promote osteoblast differentiation in aortic valve calcification. Among of them, the new molecular mechanism proposes that lncRNAs act as 'sponges' to miRNAs to manipulate the expression and function of miRNAs received lots of attention $(35,36)$. The role and molecular mechanism of miRNAs in cardiac development and diseases is been fairly well understood. Nevertheless, with the growing exploration of lncRNA function, the role of miRNAs has become a novel concern again. Though bioinformatics analysis we identified a possible relationship between TUG1 and miR-29c with starBase v2.0. Given the important role of miR-29c in fibrosis and verified the binding sites of TUG1 and miR-29c in our experiment, we further demonstrate TUG1 function as miR-29c sponge to further regulate the cardiac fibrosis in hypoxia.

The miRNA-29 s family, which targets proteins of the EMC or those related to fibrosis (7), is thought to play an critical role in multiple organic fibrosis. miRNA-29c is downregulated in renal or pulmonary hypoxia and is negatively correlated with fibrotic aggregation $(22,37)$. Our data, along with previous findings, showed a strong relationship among TUG1, miR-29c, hypoxia and fibrosis. It is reported that COLI is a target gene of miR-29c and that $\alpha$-SMA is also regulated by miR-29c $(22,38)$. The excessively activated secretion and $\alpha$-SMA expression could be signed as the fibroblast-myofibroblasts transformation. In this present study, we confirmed miR-29c ameliorated the FMT and the influence of TUG1 on miR-29c both in normxia and hypoxia. Thus, TUG1 might regulate the FMT in hypoxia by sponging miR-29c.

In conclusion, we demonstrated a novel regulator of cardiac fibrosis induced by chronic hypoxia and a TUG1-miR-29c-mediated mechanism underlying the FMT evoked by chronic hypoxia. We propose that under chronic hypoxia conditions, TUG1 might contribute to the cardiac fibroblast transition to myofibroblasts by reducing miR-29c via a RNA 'sponge' function, with more collagen accumulation and $\alpha$-SMA expression further aggregating cardiac fibrosis under hypoxia conditions. Therefore, a more thorough understanding of the precise molecular relationship between TUG1 and miR-29c might support a novel therapeutic intervention for chronic hypoxia-induced cardiac fibrosis.

\section{Acknowledgements}

Not applicable.

\section{Funding}

The present study was supported by a grant from The National Natural Science Foundation of China (grant nos. 81270228 and 81471480).

\section{Availability of data and materials}

The datasets used and/or analyzed during the present study are available from the corresponding author on reasonable request.

\section{Authors' contributions}

YZ performed the experiment and was a major contributor in writing the manuscript. YZ and ZF analyzed data. ZJ and YX proposed the idea of this work and supported the access to use the foundation for the research. All authors read and approved the final manuscript.

\section{Ethics approval and consent to participate}

The present study, including all sample collection procedures for human and animal studies, were approved by the Ethics Committee of Xinqiao Hospital (Chongqing, China). All participants provided written informed consent.

\section{Patient consent for publication}

All participants provided written informed consent.

\section{Competing interests}

The authors declare that they have no competing interests.

\section{References}

1. Chowdhury UK, Sathia S, Ray R, Singh R, Pradeep KK and Venugopal P: Histopathology of the right ventricular outflow tract and its relationship to clinical outcomes and arrhythmias in patients with tetralogy of Fallot. J Thorac Cardiovasc Surg 132: 270-277, 2006.

2. Higgins DF, Kimura K, IwanoM and Haase VH: Hypoxia-inducible factor signaling in the development of tissue fibrosis. Cell Cycle 7: 1128-1132, 2008

3. Fan D, Takawale A, Lee J and Kassiri Z: Cardiac fibroblasts, fibrosis and extracellular matrix remodeling in heart disease. Fibrogenesis Tissue Repair 5: 15, 2012.

4. Greco S, Gorospe M and MartelliF: Noncoding RNA in age-related cardiovascular diseases. J Mol Cell Cardiol 83: 142-155, 2015.

5. Condorelli G, Latronico MV and Cavarretta E: microRNAs in cardiovascular diseases: Current knowledge and the road ahead. J Am Coll Cardiol 63: 2177-2187, 2014.

6. Smith T, Rajakaruna C, Caputo M and Emanueli C: MicroRNAs in congenital heart disease. Ann Transl Med 3: 333, 2015. 
7. van Rooij E, Sutherland LB, Thatcher JE, DiMaio JM, Naseem RH, Marshall WS, Hill JA and Olson EN: Dysregulation of microRNAs after myocardial infarction reveals a role of miR-29 in cardiac fibrosis. Proc Natl Acad Sci USA 105: 13027-13032, 2008.

8. Archer K, Broskova Z, Bayoumi AS, Teoh JP, Davila A, Tang Y, $\mathrm{Su} \mathrm{H}$ and $\mathrm{Kim}$ IM: Long non-coding RNAs as master regulators in cardiovascular diseases. Int J Mol Sci 16: 23651-23667, 2015.

9. Schonrock N, Harvey RP and Mattick JS: Long noncoding RNAs in cardiac development and pathophysiology. Circ Res 111 $1349-1362,2012$

10. Esteller M: Non-coding RNAs in human disease. Nat Rev Genet 12: 861-874, 2011.

11. Wang K, Liu F, Zhou LY, Long B, Yuan SM, Wang Y, Liu CY, Sun T, Zhang XJ and Li PF: The long noncoding RNA CHRF regulates cardiac hypertrophy by targeting miR-489. Circ Res 114: 1377-1388, 2014.

12. Watson CJ, Collier P, Tea I, Neary R, Watson JA, Robinson C, Phelan D, Ledwidge MT, McDonald KM, McCann A, et al: Hypoxia-induced epigenetic modifications are associated with cardiac tissue fibrosis and the development of a myofibroblast-like phenotype. Hum Mol Genet 23: 2176-2188, 2014.

13. Chang YN, Zhang K, Hu ZM, Qi HX, Shi ZM, Han XH, Han YW and Hong W: Hypoxia-regulated lncRNAs in cancer. Gene 575: $1-8,2016$.

14. Xie S, Chen H, Li F, Wang S and Guo J: Hypoxia-induced microRNA-155 promotes fibrosis in proximal tubule cells. Mol Med Rep 11: 4555-4560, 2015.

15. Liu M, Liu L, Bai M, Zhang L, Ma F, Yang X and Sun S: Hypoxia-induced activation of Twist/miR-214/E-cadherin axis promotes renal tubular epithelial cell mesenchymal transition and renal fibrosis. Biochem Biophys Res Commun 495: 2324-2330, 2018.

16. Zhang QQ, Xu MY, Qu Y, Hu JJ, Li ZH, Zhang QD and Lu LG: TET3 mediates the activation of human hepatic stellate cells via modulating the expression of long non-coding RNA HIF1A-AS1. Int J Clin Exp Pathol 7: 7744-7751, 2014

17. Liu Y, Li Y, Xu Q, Yao W, Wu Q, Yuan J, Yan W, Xu T, Ji X and Ni C: Long non-coding RNA-ATB promotes EMT during silica-induced pulmonary fibrosis by competitively binding miR-200c. Biochim Biophys Acta 1864: 420-431, 2018

18. Nagpal V, Rai R, Place AT, Murphy SB, Verma SK, Ghosh AK and Vaughan DE: MiR-125b is critical for fibroblast-to-myofibroblast transition and cardiac fibrosis. Circulation 133: 291-301, 2016.

19. Zhang J, Chang JJ, Xu F, Ma XJ, Wu Y, Li WC, Wang HJ, Huang GY and Ma D: MicroRNA deregulation in right ventricular outflow tract myocardium in nonsyndromic tetralogy of fallot. Can J Cardiol 29: 1695-1703, 2013.

20. Poliseno L, Salmena L, Zhang J, Carver B, Haveman WJ and Pandolfi PP: A coding-independent function of gene and pseudogene mRNAs regulates tumour biology. Nature 465: 1033-1038, 2010.

21. Roncarati R, Viviani Anselmi C, Losi MA, Papa L, Cavarretta E, Da Costa Martins P, Contaldi C, Saccani Jotti G, Franzone A Galastri L, et al: Circulating miR-29a, among other up-regulated microRNAs, is the only biomarker for both hypertrophy and fibrosis in patients with hypertrophic cardiomyopathy. J Am Coll Cardiol 63: 920-927, 2014.

22. Zhang HW, Wang EW, Li LX, Yi SH, Li LC, Xu FL, Wang DL, Wu YZ and Nian WQ: A regulatory loop involving miR-29c and Sp1 elevates the TGF- $\beta 1$ mediated epithelial-to-mesenchymal transition in lung cancer. Oncotarget 7: 85905-85916, 2016.

23. Wang JH, Zhao L, Pan X, Chen NN, Chen J, Gong QL, Su F, Yan J, Zhang Y and Zhang SH: Hypoxia-stimulated cardiac fibroblast production of IL-6 promotes myocardial fibrosis via the TGF- $\beta 1$ signaling pathway. Lab Invest 96: 839-852, 2016.
24. Pedroza M, Le TT, Lewis K, Karmouty-Quintana H, To S, George AT, Blackburn MR, Tweardy DJ and Agarwal SK: STAT-3 contributes to pulmonary fibrosis through epithelial injury and fibroblast-myofibroblast differentiation. FASEB J 30: 129-140, 2016.

25. Baumann B, Hayashida T, Liang X and Schnaper HW: Hypoxia-inducible factor- $1 \alpha$ promotes glomerulosclerosis and regulates COL1A2 expression through interactions with Smad3. Kidney Int 90: 797-808, 2016.

26. Klattenhoff CA, Scheuermann JC, Surface LE, Bradley RK, Fields PA, Steinhauser ML, Ding H, Butty VL, Torrey L, Haas S, et al: Braveheart, a long noncoding RNA required for cardiovascular lineage commitment. Cell 152: 570-583, 2013

27. Kumarswamy R, Bauters C, Volkmann I, Maury F, Fetisch J, Holzmann A, Lemesle G, de Groote P, Pinet F and Thum T: Circulating long noncoding RNA, LIPCAR, predicts survival in patients with heart failure. Circ Res 114: 1569-1575, 2014.

28. Young TL, Matsuda T and Cepko CL: The noncoding RNA taurine upregulated gene 1 is required for differentiation of the murine retina. Curr Biol 15: 501-512, 2005.

29. Liang S, Zhang S, Wang P, Yang C, Shang C, Yang J and Wang J: LncRNA, TUG1 regulates the oral squamous cell carcinoma progression possibly via interacting with Wnt/ $\beta$-catenin signaling. Gene 608: 49-57, 2017.

30. Zhang L, Cheng H, Yue Y, Li S, Zhang D and He R: TUG1 knockdown ameliorates atherosclerosis via up-regulating the expression of miR-133a target gene FGF1. Cardiovasc Pathol 33: 6-15, 2018.

31. Michalik KM, You X, Manavski Y, Doddaballapur A, Zörnig M, Braun T, John D, Ponomareva Y, Chen W, Uchida S, et al: Long noncoding RNA MALAT1 regulates endothelial cell function and vessel growth. Circ Res 114: 1389-1397, 2014.

32. He Q, Yang S, Gu X, Li M, Wang C and Wei F: Long noncoding RNA TUG1 facilitates osteogenic differentiation of periodontal ligament stem cells via interacting with Lin28A. Cell Death Dis 9: 455, 2018.

33. Zhang E, He X, Yin D, Han L, Qiu M, Xu T, Xia R, Xu L, Yin R and De W: Increased expression of long noncoding RNA TUG1 predicts a poor prognosis of gastric cancer and regulates cell proliferation by epigenetically silencing of $\mathrm{p} 57$. Cell Death Dis 7: e2109, 2016.

34. Yu C, Li L, Xie F, et al: LncRNA TUG1 sponges miR-204-5p to promote osteoblast differentiation through upregulating Runx2 in aortic valve calcification. Cardiovasc Res 114: 168-179, 2018.

35. Cesana M, Cacchiarelli D, Legnini I, Santini T, Sthandier O, Chinappi M, Tramontano A and Bozzoni I: A long noncoding RNA controls muscle differentiation by functioning as a competing endogenous RNA. Cell 147: 358-369, 2011.

36. Tang W, Shen Z, Guo J and Sun S: Screening of long non-coding RNA and TUG1 inhibits proliferation with TGF- $\beta$ induction in patients with COPD. Int J Chron Obstruct Pulmon Dis 11: 2951-2964, 2016.

37. Fang Y, Yu X, Liu Y, Kriegel AJ, Heng Y, Xu X, Liang M and Ding $X$ : miR-29c is downregulated in renal interstitial fibrosis in humans and rats and restored by HIF- $\alpha$ activation. Am J Physiol Renal Physiol 304: F1274-F1282, 2013.

38. Khalil W, Xia H, Bodempudi V, Kahm J, Hergert P, Smith K, Peterson M, Parker M, Herrera J, Bitterman PB and Henke CA: Pathologic regulation of collagen I by an aberrant protein phosphatase 2A/histone deacetylase C4/MicroRNA-29 signal axis in idiopathic pulmonary fibrosis fibroblasts. Am J Respir Cell Mol Biol 53: 391-399, 2015. 